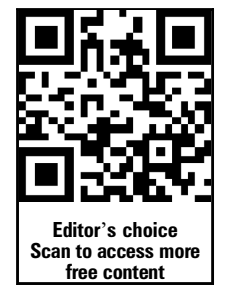

Department of Community Emergency Health and Paramedic Practice, Monash University, Melbourne, Victoria, Australia

${ }^{2}$ Department of Epidemiology and Preventive Medicine,

Monash University, Melbourne, Victoria, Australia

${ }^{3}$ Ambulance Victoria,

Melbourne, Victoria, Australia

${ }^{4}$ The Alfred Hospital,

Melbourne, Victoria, Australia

Correspondence to Dr Paul A Jennings,

Department of Community Emergency Health and

Paramedic Practice, Monash University, Level 5, The Alfred Centre, 99 Commercial Road, Melbourne, VIC 3004 Australia;

paul.jennings@monash.edu

Received 20 May 2013 Revised 24 June 2013

Accepted 27 June 2013 Published Online First 13 July 2013

\section{SLinked}

http://dx.doi.org/10.1136/ emermed-2014-203675

CrossMark

To cite: Jennings $P A$, Cameron $\mathrm{P}$, Bernard $\mathrm{S}$, et al. Emerg Med J 2014;31: 840-843.

\title{
Long-term pain prevalence and health-related quality of life outcomes for patients enrolled in a ketamine versus morphine for prehospital traumatic pain randomised controlled trial
}

\author{
Paul A Jennings, ${ }^{1,2,3}$ Peter Cameron, ${ }^{2}$ Stephen Bernard, ${ }^{2,3,4}$ Tony Walker, $^{3}$ \\ Damien Jolley, ${ }^{2}$ Mark Fitzgerald, ${ }^{3,4}$ Kevin Masci $^{3}$
}

\begin{abstract}
Introduction Improved early pain control may affect the longer-term prevalence of persistent pain. In a previous randomised, controlled trial, we found that the administration of ketamine on hospital arrival decreased pain scores to a greater extent than morphine alone in patients with prehospital traumatic pain. In this followup study, we sought to determine the prevalence of persistent pain and whether there were differences in patients who received ketamine or morphine.

Methods This study was a long-term follow-up study of the prehospital, prospective, randomised, controlled, open-label study comparing ketamine with morphine in patients with trauma and a verbal pain score of $>5$ after $5 \mathrm{mg}$ intravenous morphine. Patients were followed-up by telephone 6-12 months after enrolment, and a questionnaire including the SF-36 (V.2) health-related quality of life survey and the Verbal Numerical Rating Scale for pain was administered.
\end{abstract}

Results A total of $97 / 135(72 \%)$ patients were able to be followed-up 6-12 months after enrolment between July 2008 and July 2010. Overall, 44/97 (45\%) participants reported persistent pain related to their injury, with 3/97 (3\%) reporting persistent severe pain. The prevalence of persistent pain was the same between study groups (22/50 (44\%) for the ketamine group vs $22 / 47$ (46\%) for the morphine group). There was no difference in the SF-36 scores between study arms. Conclusions There is a high incidence of persistent pain after traumatic injury, even in patients with relatively minor severity of injury. Although decreased pain scores at hospital arrival are achieved with ketamine compared with morphine, this difference does not affect the prevalence of persistent pain or healthrelated quality of life 6 months after injury. Further larger studies are required to confirm this finding.

Trial Registration Number Australian and New Zealand Clinical Trials Registry (ACTRN12607000441415).

\section{INTRODUCTION}

Acute pain is a common presentation to healthcare clinicians, ${ }^{1-4}$ and is a significant contributor to disability many months, or years, after injury. The effective management of pain is obviously of critical importance in the short term, yet the effectiveness of early pain management may also be associated with the likelihood of persistent pain syndromes and the pain-related anxiety and distress that follow. ${ }^{5-8}$ One of the theories postulated for the progression of acute to persistent pain is central sensitisation, whereby nociceptive neurons increase their response to non-painful stimuli, develop spontaneous activity, and broaden the area of the body that is involved with the pain over time. ${ }^{9}$ Persistent pain significantly affects people's physical and mental health, ${ }^{5-8}$ and can delay functional recovery after traumatic injury. ${ }^{10}{ }^{11}$ The prevalence of persistent pain after injury has been reported to be $11-67 \%,{ }^{12-15}$ and it adversely affects health-related quality of life.

Most acute traumatic pain management studies focus on efficient reduction of pain during the episode of care, but few studies follow patients over the medium to long term after injury. The aim of this study was to determine the prevalence of persistent pain and self-reported health-related quality of life outcomes 6-12 months after injury and enrolment in a prospective randomised controlled study that compared the effectiveness of morphine with morphine and ketamine in patients with moderate to severe traumatic pain before arrival at hospital.

\section{METHODS}

The setting and study design of the morphine and ketamine versus morphine alone randomised controlled trial has been previously described. ${ }^{16}$ Briefly, patients with trauma and a verbal pain score of $>5$ after $5 \mathrm{mg}$ intravenous morphine were eligible for enrolment. Those with any of the following were excluded: known allergy to ketamine hydrochloride or morphine sulfate, pregnant or lactating women, ischaemic chest pain, acute pulmonary oedema, and/or severe hypertension (systolic blood pressure $>180 \mathrm{~mm} \mathrm{Hg}$ ), head injury with history of loss of consciousness or Glasgow Coma Score $<15$, no venous access available, or presumed intoxication with alcohol or illicit substance. Patients allocated to ketamine received a bolus of $20 \mathrm{mg}$ followed by $10 \mathrm{mg}$ every $3 \mathrm{~min}$. Patients allocated to morphine alone received up to $5 \mathrm{mg}$ intravenously every 5 min until pain free (standard care). Pain scores were measured at baseline and at hospital arrival.

Patients were then followed-up via a telephone interview 6-12 months after enrolment into the study. Two instruments were administered to participants enrolled in this study to measure both the presence of persistent pain and health-related quality of life: the Short Form 36 V.2 Health Survey (SF-36; QualityMetric, Lincoln, Rhode Island , USA ${ }^{17}$ and a global pain numerical rating 
score. The SF-36 was divided into two aggregate summary measures: the Physical Component Summary (PCS) and the Mental Component Summary (MCS). ${ }^{17}$ The global pain measure used the Verbal Numerical Rating Scale (VNRS) ${ }^{18}$ and reported the patient's self-rated current pain at the time of the interview. Patients were asked to rate their current pain intensity using an 11 point scale $0-10$ ( 0 being 'no pain' and 10 being 'worst pain ever experienced').

Participants were eligible if they had been recruited for the ketamine study. ${ }^{16}$ Each participant was contacted by telephone 1 month after enrolment to obtain consent for access to their medical record from the receiving hospital and for a follow-up telephone call for the purpose of conducting the questionnaire. Participants were contacted via the landline or mobile phone number recorded on the case report form at the time of enrolment. If the participant was not contactable via the phone numbers provided at enrolment (ie, phone numbers disconnected, not responding to messages left on answering machines, no answer, and wrong numbers) after at least five attempts, additional sources were used to identify current contact details. These sources included the Whitepages, the patient's medical discharge summary (where available), and the ambulance service patient database. The telephone interview took $\sim 20 \mathrm{~min}$ to complete, and each participant was offered the opportunity to ask questions about the study and their participation. Several trained interviewers conducted the telephone interviews, and each was blinded to the trial arm allocation of the participant at the time of the interview. Owing to the method of SF-36 administration (telephone interview), there were no missing data. Only one person contacted for follow-up interview refused to take part in the SF-36.

\section{Statistical analysis}

The sample size calculation has been provided previously, ${ }^{16}$ and was powered to detect a difference in the primary outcome measure (change in VNRS score), not the secondary outcome measures, which include the long-term outcomes. Transformation of raw scores was performed using Stata V.11 using the published Australian and New Zealand population norms. ${ }^{19}$ The baseline characteristics and SF-36 summary and scale scores were compared using the two-sample $t$ test for continuous variables. The global pain scale scores were compared using the two-sample Wilcoxon rank-sum test, as these data were not normally distributed. Treatment effects were tested at a two-sided significance level of 0.05 .

\section{RESULTS}

A total of 136 participants with moderate to severe traumatic pain were enrolled between December 2007 and July 2010 (a 30-month period). Follow-up of participants continued until December 2010. One person withdrew consent to participate in the trial, leaving 135 participants eligible for analysis. Sixty-five (48\%) participants were randomly assigned to the morphine-only group (M group) and $70(52 \%)$ to the ketamine plus morphine group ( $\mathrm{K}+\mathrm{M}$ group). The baseline characteristics were similar between the two groups and are shown in table 1.

Study participants could be followed-up in $72 \%$ of cases (97/ $135)$. Thirty-seven participants $(27 \%)$ were unable to be contacted by phone, and one participant $(1 \%)$ refused to participate in the interview. Of those who were available for follow-up, $48 \%$ (47/97) had been enrolled in the ketamine arm and 52\% $(50 / 97)$ in the morphine arm.
Table 1 Demographic data and injury characteristics of patients

\begin{tabular}{|c|c|c|}
\hline Characteristic & $\begin{array}{l}K+M \text { group } \\
(n=70)\end{array}$ & $\begin{array}{l}M \text { group } \\
(n=65)\end{array}$ \\
\hline \multicolumn{3}{|l|}{ Sex (n (\%)) } \\
\hline Male & $45(64)$ & $38(58)$ \\
\hline Female & $25(36)$ & $27(42)$ \\
\hline \multicolumn{3}{|l|}{ Age (years) } \\
\hline Mean (SD) & $44.2(20.3)$ & $48.5(20.5)$ \\
\hline Median & 41 & 45 \\
\hline Min, max & 18,90 & 18,96 \\
\hline \multicolumn{3}{|l|}{ Case nature (N (\%)) } \\
\hline Extremity fracture & $26(37)$ & $29(45)$ \\
\hline Soft tissue injury & $17(24)$ & $15(23)$ \\
\hline Fracture, other & $14(20)$ & $13(20)$ \\
\hline Dislocation & $11(16)$ & $7(11)$ \\
\hline Burn & $2(3)$ & $1(1)$ \\
\hline \multicolumn{3}{|l|}{ Injury Severity Score* } \\
\hline Mean (SD) & $4.3(3.5)$ & $4.9(4.5)$ \\
\hline Median & 4 & 4 \\
\hline Min, max & 0,13 & 0,22 \\
\hline \multicolumn{3}{|l|}{ Initial pain score } \\
\hline Mean (SD) & $7.5(1.7)$ & $7.4(1.8)$ \\
\hline Median & 7.5 & 7 \\
\hline Min, $\max$ & 5,10 & 5,10 \\
\hline \multicolumn{3}{|c|}{ Number of patients to whom methoxyflurane was administered } \\
\hline Frequency (\%) & $48(68.6)$ & $40(61.5)$ \\
\hline \multicolumn{3}{|c|}{ Dose of methoxyflurane administered $(\mathrm{mL})$} \\
\hline Mean (SD) & $3.1(0.4)$ & $3.4(1.0)$ \\
\hline Median & 3 & 3 \\
\hline Min, max & 0,6 & 0,6 \\
\hline $\begin{array}{l}\text { Dose of trial drug administered after } \\
\text { randomisation }(\mathrm{mg})\end{array}$ & Ketamine & Morphine \\
\hline Mean (SD) & $40.6(25.0)$ & $14.4(9.4)$ \\
\hline Median & 35 & 15 \\
\hline Min, max & 10,120 & $2.5,60$ \\
\hline \multicolumn{3}{|l|}{ Prehospital time (min) } \\
\hline Mean (SD) & $52.4(20.8)$ & $50.5(21.0)$ \\
\hline Median & 49.5 & 45 \\
\hline Min, $\max$ & 20,103 & 18,123 \\
\hline
\end{tabular}

*Missing data $\mathrm{n}=6$ ( 3 from each group).

$\mathrm{K}+\mathrm{M}$ group, ketamine plus morphine group; $\mathrm{M}$ group, morphine-only group.

\section{Responders vs non-responders}

Non-responders and responders were very similar with respect to all characteristics, except that non-responders were less likely to be male $(50 \%$ vs $66 \%)$ and were slightly more severely injured (mean Injury Severity Score 6.2 vs 4.0 ).

\section{Outcomes at follow-up}

Table 2 summarises PCS and MCS scores of the 97 participants who were followed-up by participant characteristic.

The overall mean PCS and MCS summary measure scores were the same for the two study groups (table 3).

Overall, 45\% (44/97) of participants reported persistent pain related to their injury, with $3 \%$ (3/97) reporting persistent severe (VNRS $>7$ ) pain. The prevalence of persistent pain was approximately the same in the two study groups $(\mathrm{K}+\mathrm{M}$ group: 44\% (22/50); M group: 46\% (22/47)). The self-reported global median pain score at follow-up was no different between the $\mathrm{K}+\mathrm{M}$ group (0 (IQR $0-2)$ ) and the $\mathrm{M}$ group (0 (IQR $0-3)$ ) (Wilcoxon $\mathrm{p}$ value $=0.44)$. 
Table 2 Physical Component Summary (PCS) score and Mental Component Summary (MCS) score by participant characteristic

\begin{tabular}{|c|c|c|c|c|c|c|c|}
\hline \multirow[b]{2}{*}{ Characteristic } & \multirow[b]{2}{*}{$\mathrm{N}$} & \multicolumn{3}{|l|}{ PCS } & \multicolumn{3}{|l|}{ MCS } \\
\hline & & $\mathrm{K}+\mathrm{M}$ group & $M$ group & p Value* & $\mathrm{K}+\mathrm{M}$ group & $M$ group & p Value* \\
\hline \multicolumn{8}{|l|}{ Sex } \\
\hline Male & 64 & 49.5 (11.6) & $49.0(12.0)$ & 0.88 & $50.8(11.0)$ & $50.8(12.1)$ & 0.99 \\
\hline Female & 33 & $48.0(10.3)$ & $45.8(8.2)$ & 0.51 & $48.3(14.0)$ & 48.5 (15.4) & 0.97 \\
\hline \multicolumn{8}{|l|}{ Case nature } \\
\hline Extremity fracture & 33 & $47.1(10.6)$ & $48.7(7.8)$ & 0.61 & $47.2(14.4)$ & $52.3(12.2)$ & 0.28 \\
\hline Soft tissue injury & 26 & $52.5(7.3)$ & $48.2(18.0)$ & 0.41 & $52.0(9.8)$ & $48.9(17.1)$ & 0.56 \\
\hline Fracture, other & 21 & $47.8(12.3)$ & $45.6(8.9)$ & 0.64 & 49.5 (13.5) & $47.2(16.0)$ & 0.73 \\
\hline Dislocation & 15 & $45.7(16.3)$ & $47.0(9.4)$ & 0.86 & $51.0(11.0)$ & $48.7(6.1)$ & 0.63 \\
\hline Burn & 2 & $58.4(0)$ & $59.6(0)$ & NA & $57.4(0)$ & $52.6(0)$ & NA \\
\hline \multicolumn{8}{|l|}{ Injury Severity Score } \\
\hline $0-4$ & 77 & 49.1 (10.9) & 47.8 (11.5) & 0.61 & $49.2(12.6)$ & $49.4(14.0)$ & 0.94 \\
\hline $5-14$ & 14 & $51.3(8.7)$ & $50.9(8.3)$ & 0.93 & $55.2(8.1)$ & $56.4(5.8)$ & 0.76 \\
\hline $15+$ & 6 & $37.8(25.5)$ & $44.9(9.7)$ & 0.62 & $46.4(13.2)$ & $45.5(12.1)$ & 0.94 \\
\hline \multicolumn{8}{|l|}{ Initial pain score } \\
\hline $5-7$ & 51 & $51.4(6.8)$ & $48.8(10.0)$ & 0.28 & $52.0(10.6)$ & $51.3(11.6)$ & 0.82 \\
\hline $8-10$ & 46 & $46.1(14.4)$ & $47.0(12.0)$ & 0.82 & 47.7 (13.4) & $48.6(14.9)$ & 0.84 \\
\hline \multicolumn{8}{|l|}{ Final pain score } \\
\hline $0-4$ & 68 & $49.1(10.7)$ & $49.2(10.0)$ & 0.98 & $50.0(12.4)$ & $49.6(12.1)$ & 0.92 \\
\hline $5-7$ & 22 & $56.2(2.1)$ & 44.7 (12.5) & 0.08 & $53.3(7.9)$ & $48.3(15.6)$ & 0.54 \\
\hline $8-10$ & 7 & $30.9(15.6)$ & $53.4(6.2)$ & 0.03 & $45.3(11.6)$ & $57.7(6.8)$ & 0.12 \\
\hline \multicolumn{8}{|l|}{ Prehospital time (min) } \\
\hline$<60 \min$ & 68 & $48.4(11.4)$ & $48.6(11.9)$ & 0.94 & $50.5(12.9)$ & $48.6(13.8)$ & 0.57 \\
\hline$\geq 60 \min$ & 29 & $50.1(10.8)$ & $45.9(7.1)$ & 0.25 & $49.2(10.2)$ & $53.9(10.8)$ & 0.24 \\
\hline
\end{tabular}

\section{DISCUSSION}

There was no clinically significant difference in the long-term follow-up between study groups with respect to the summary or global pain score. Even so, this study is important, as it provides information about the long-term evaluation of management of acute pain. Most studies focus only on the immediate effect of the intervention.

There was no difference in the median self-reported global pain scale between the two study groups; however, $45 \%$ of participants reported persistent pain as a result of their injury. This finding is particularly important given the relatively minor nature of the injuries sustained, most being isolated musculoskeletal trauma. Early, effective pain management is important for several reasons. First, pain relief after injury is important for humanitarian reasons. Perhaps of equal, or arguably more, importance, earlier and more effective treatment of acute pain may reduce or even terminate the progression from acute to persistent pain. ${ }^{10} 13$ Effective pain management in both the emergency medical services (EMS) and emergency department setting may play a role in reducing the likelihood of chronic

Table 3 Summary measures by study group

\begin{tabular}{llll}
\hline Characteristic & $\begin{array}{l}\text { K+M group } \\
(\mathrm{N}=50)\end{array}$ & $\begin{array}{l}\text { M group } \\
(\mathrm{N}=47)\end{array}$ & p Value* \\
\hline Physical Component Summary & $49.0(11.1)$ & $47.9(10.9)$ & 0.64 \\
Mental Component Summary & $50.0(12.0)$ & $50.0(13.2)$ & 0.98 \\
\hline Values aremean (SD). & & & \\
$\quad$ & & \\
KTwo-sample t test. & &
\end{tabular}

pain syndromes and pain-related anxiety and distress after the acute phase. ${ }^{5-8}$ The phenomenon of the progression of acute pain to chronic pain syndromes is also seen in surgery, where there is strong evidence that some early anaesthetic/analgesic interventions reduce the incidence of chronic pain after surgery. ${ }^{20}$ Furthermore, persistent pain is a substantial contributor to the burden of disease after injury, significantly impacting on people's physical and mental health, ${ }^{5-8}$ and can delay functional recovery after traumatic injury. ${ }^{10} 11$

Future pain management research should also aim to follow participants over time to evaluate the effect the interventions have on long-term physical and mental health outcomes and the association of acute pain characteristics and management with the development of persistent pain.

\section{Limitations}

A limitation to the analysis of long-term global pain and quality of life of the participants in this study was the loss to follow-up. Follow-up was possible in $72 \%(97 / 135)$ of participants, and it is possible that the inability to locate all study participants may affect the validity of the study's secondary end point-longterm health-related outcome. There was, however, little difference in the characteristics profile of those who responded and those who did not respond. Furthermore, the number of patients included in this phase of the study was moderate, and therefore the power to identify a difference between groups, if one exists, may have been limited. We did not have the 'time to treatment' interval available to us, and we feel that this may have been an important covariate and should be collected and considered for future studies in this area. 
Finally, it was not possible to interview all study participants at the same time period after their enrolment. While it was intended to conduct interviews 6 months after enrolment, a number of participants were unable to be contacted until up to 12 months after recruitment.

\section{CONCLUSION}

There is a high incidence of persistent pain after traumatic injury, even in patients with relatively minor severity of injury. There was no difference in persistent pain prevalence, global median pain intensity or SF-36 summary measures in this prehospital trauma cohort. We feel this study, despite being moderate in size, provides valuable information for researchers aiming to undertake further, larger-scale research in this area of persistent pain mechanisms and aetiology. Furthermore, clinical trials that examine acute pain management interventions should include in their study design medium- to long-term follow-up of health-related quality of life and prevalence of persistent pain.

Acknowledgements The authors would like to acknowledge the contribution of the paramedics from Ambulance Victoria who assisted by enrolling participants and the submission of data.

Contributors PAJ, PC, and SB conceived the study, and PAJ, PC, SB, TW, MF and $\mathrm{KM}$ designed the trial. PAJ obtained research funding through a TAC research fellowship. PAJ, PC, SB, TW and KM supervised the conduct of the trial and data collection. PAJ undertook recruitment of participating sites and patients and managed the data, including quality control. DJ provided statistical advice and analysed the data. TW chaired the trial steering committee. PAJ drafted the manuscript, and all authors contributed substantially to its revision. PAJ takes responsibility for the paper as a whole.

Funding Transport Accident Commission (TAC) Health Research Fellowship.

Competing interests None.

Ethics approval Monash University Human Research Ethics Committee, Melbourne, Australia.

Provenance and peer review Not commissioned; externally peer reviewed.

\section{REFERENCES}

1 Alonso-Serra HM, Wesley K, National Association of EMSPSaCPC. Prehospital pain management. [see comment]. Prehosp Emerg Care 2003;7:482-8.
2 Hennes H, Kim MK, Pirrallo RG. Prehospital pain management: a comparison of providers' perceptions and practices. Prehosp Emerg Care 2005;9:32-9.

3 Jennings PA, Cameron P, Bernard S. Epidemiology of prehospital pain: an opportunity for improvement. Emerg Med I 2011;28:530-1.

4 McManus JG Jr, Sallee DR Jr. Pain management in the prehospital environment. Emerg Med Clin North Am 2005;23:415-31.

5 Buckenmaier CC III, Rupprecht C, McKnight G, et al. Pain following battlefield injury and evacuation: a survey of 110 casualties from the wars in Iraq and Afghanistan. Pain Med 2009;10:1487-96.

6 Turturro MA. Pain, priorities and prehospital care. Prehosp Emerg Care 2002;6:486-8.

7 Thomas SH, Shewakramani S. Prehospital Trauma Analgesia. J Emerg Med 2008:35:47-57.

8 Weisman SJ, Bernstein B, Schechter NL. Consequences of inadequate analgesia during painful procedures in children. Arch Pediatric Adolesc Med 1998;152: 147-9.

9 Kyranou M, Puntillo K. The transition from acute to chronic pain: might intensive care unit patients be at risk? Ann Intensive Care 2012;2.

10 Castillo RC, Mackenzie EJ, Wegener ST, et al. Prevalence of chronic pain seven years following limb threatening lower extremity trauma. Pain 2006;124:321-9.

11 Mkandawire NC, Boot DA, Braithwaite IJ, et al. Musculoskeletal recovery 5 years after severe injury: long term problems are common. Injury 2002;33:111-15.

12 Hoffman JM, Pagulayan KF, Zawaideh N, et al. Understanding pain after traumatic brain injury: impact on community participation. Am J Phys Med Rehabil 2007:86:962-9.

13 Rivara FP, Mackenzie EJ, Jurkovich GJ, et al. Prevalence of pain in patients 1 year after major trauma. Arch Surg 2008;143:282-7.

14 Andrew NE, Gabbe BJ, Wolfe R, et al. Twelve-month outcomes of serious orthopaedic sport and active recreation-related injuries admitted to Level 1 trauma centers in Melbourne, Australia. Clin J Sport Med 2008;18:387-93.

15 Moore CM, Leonardi-Bee J, Moore CM, et al. The prevalence of pain and disability one year post fracture of the distal radius in a UK population: a cross sectional survey. BMC Musculoskelet Disord 2008;9:129.

16 Jennings $P$, Cameron $P$, Bernard $S$, et al. Morphine and ketamine is superior to morphine alone for out-of-hospital trauma analgesia: A randomized controlled trial. Ann Emerg Med 2012;59:497-503.

17 Marosszeky N. Australian Health Outcomes Collaboration SF-36 Health Survey (Version 1.0) Instrument Review. 2005. http://chsduoweduau/ahoc/documents/ sf36reviewpdf (accessed 10 Dec 2010).

18 Jennings PA, Cameron P, Bernard S. Measuring acute pain in the prehospital setting. Emerg Med J 2009;26:552-5.

19 Ware JE, Kosinski M, Bjorner JB, et al. User's manual for the SF-36v2 health survey. 2nd edn. Lincoln, RI: QualityMetric Incorporated, 2007.

20 Maclntyre PA, Scott DA, SA S, et al. Acute pain management: scientific evidence. 3rd edn. Australian and New Zealand College of Anaethetists and Faculty of Pain Medicine, 2010 